\title{
Possibilities and limitations of magnetic source imaging of methohexital- induced epileptiform patterns in temporal lobe epilepsy patients
}

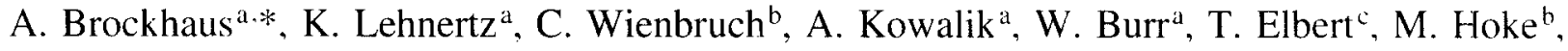 \\ C.E. Elger ${ }^{\mathrm{a}}$ \\ "Department of Epileptology. University of Bom, Sigmund-Freud-Strasse 25, 53105 Bonn. Germamy \\ 'Institute of Experimental Audioligy: University of Münster. Miunster. Germany \\ "Department of Psychology, University of Konstan-, Konstan-, Germany.
}

\begin{abstract}
The usefulness of MEG-based techniques in lateralizing and localizing the epileptogenic area was investigated in the present study. Spontaneous and methohexital-induced spikes were studied in a group of to patients with temporomesial epilepsy using a 37-channel neuromagnetometer. The accuracy of the magnetic source imaging was compared to the results of electrocorticographic (ECoG) recordings. Differcnces of drug-induced spike densities in the MEG recordings between both sides confirmed a similar lateralizing power of the MEG and ECoG recordings. Source location analyses based on a moving dipole model resp. a rotating dipole model were performed using a spherical head model. After subdivision of the volume of each patient's head. $8 \mathrm{~cm}^{3}$ cubicles containing at least 3 source locations were projected onto the individual MRI scan and resulted in source locations within or close to the presurgically defined primary epileptogenic area only in 3 of the 15 patients. Spike induction by methohexital has the advantage of shortening the recording period as compared to recordings of interictal epileptiform discharges. However, the correlation analyses of spike densities from MEG and ECoG recordings and the source location analyses from MEG recordings indicate that spikes generated in deep temporomesial structures may escape the MEG registration. 1997 Elsevier Science Ireland Ltd.
\end{abstract}

Keywords: Magnetoencephalography: Electrocorticography; Temporal lobe epilepsy: Source localization; Methohexital

\section{Introduction}

Epilepsy surgery requires the exact delineation and localization of the epileptogenic zone. To date the most reliable results are provided by electrocorticographic registration (ECoG) of seizures using subdural electrodes and/or intracerebral depth electrodes (Elger, 1992; Lüders et al., 1993). However. in order to avoid invasive diagnostic procedures. reliable techniques for non-invasive focus localization are required. Magnetoencephalography (MEG) permits the non-invasive evaluation of magnetic fields produced by neuronal activity. Concerning focus localization, the MEG offers the following advantages compared to the EEG:

1. The different conductive properties of skull, scalp, cere-

\footnotetext{
* Corresponding author. Tel.: +49 228 2875864; fax: +492282876294 :
} e-mail: anke(a) jersey.meb.uni-bonn.ce. brospinal fluid and brain must be taken into account for EEG-based source-modeling, whereas it is sufficient to model the local head shape in the case of magnetic source imaging.

2. No reference is needed for the MEG recordings, whereas for EEG recordings always a reference electrode is necessary'.

Despite these advantages, the value of $M E G$ recordings for the localization of the primary epileptogenic area is still controversially discussed (Stefan et al.. 1990), 1994; Tiihonen et al., 1990; Nakasato et al.. 1994; Pateau et al.. 1994; Ebersole et al., 1995; Smith et al., 1995). One problem consists in recording seizures in the MEG because movement artifacts during seizures render reliable MEG recordings almost impossible. Therefore, most authors have investigated interictal spike activity in the MEG. However, interictal spike activity is considered to be of minor value as 
far as the localization of the primary epileptogenic area is concerned. According to Lüders et al. (1993), interictal spike activity mainly reflects the irritative zone. which in general is larger than the primary epileptogenic zone. The area of seizure onset corresponds best to the primary epileptogenic zone which has to be resected in order to achieve seizure free outcome after surgery (Elger, 1992; Lüders et al., 1993). According to the concept of an irritative and a primary epileptogenic zone, the value of interictal spikes in EEG and ECoG recordings concerning focus localization is well defined, but the significance of interictal spikes in the MEG recordings with respect to the irritative and the primary epileptogenic zone has not been established until now. Furthermore, in most epilepsy patients interictal spikes represent an inconstant and infrequent phenomenon. Therefore, in order to record a sufficient number of spikes, longlasting recording periods are required. The necessity to keep patients motionless during the MEG recordings causes additional problems. To shorten the recording period, techniques that increase the frequency of spikes would be useful.

Barbiturates are known to have activating effects on epileptiform discharges. Short-acting barbiturates are used to identify the epileptogenic focus during preoperative evaluation for epilepsy surgery (Fuster et al., 1948; Pampiglione, 1952; Bickford et al., 1953; Brazier, 1969; Harris and Paul, 1969; Wilder, 1971; Lieb et al., 1974). Many reports about the effects of the different barbiturates as methohexital (Musella et al., 1969; Gumpert and Paul, 1971; Celcsia and Paulsen, 1972; Ford et al., 1982; Wyler et al., 1987; asley et al., 1989; Hufnagel et al., 1992), amobarbital (Aasley et al., 1989; Kiersey et al., 1951; Hufnagel et al., 1990a), thiopental (Sperling et al., 1986; Lieb et al., 1989; Dasheiff and Kofke, 1993; Brockhaus et al., 1995) and the narcotic propofol (Hazeaux et al., 1987; Hufnagel et al., 1990b) have shown that different short-acting barbiturates and narcotics have distinct activative and suppressive effects on epileptiform discharges in EEG and ECoG recordings. Methohexital is known to be one of the barbiturates with a high potency to activate epileptiform discharges. Hufnagel et al. (1992) showed that methohexital increases the spike density and, furthermore, activates another quality of epileptiform pattern described as spike. burst suppression (SBS) pattern which is characterized by a high amplitude spike hurst followed by a suppression in the EEG/ECoG consisting of isoelectrical activity or subdelta background activity. Spikes and SBS pattern induced by methohexital in the ECoG recorded with temporal subdural strip electrodes are known to be of good localizing value concerning the primary epileptogenic zone (Wyler et al., 1987; Hufnagel et al., 1992). Furthermore, the results concerning focus localization by methohexital activation of epileptiform discharges are reproducible. Kowalik et al. (1997) performed successive methohexital narcoses in a group of temporal lobe epilepsy patients and showed that the brain region where a SBS pattern was activated remained at the same location in more than $90 \%$ of the patients. Additionally, changes of serum levels of the anticonvulsive drugs did not influence the results.

The high potency of methohexital to activate spikes should be useful to increase spike activity during MEG recordings. Besides of a reduced recording period, the short narcosis induced by methohexital should provide recordings without movement artifacts due to reduced motor activity. Because of the good localizing value of methohexital-induced epileptiform discharges in ECoG recordings with subdural strip electrodes, it can be expected that MEG recordings of methohexital-induced spikes provide more precise data concerning the epileptogenic zone than solely measuring interictal spike activity. To our knowledge, barbiturate-induced epileptiform activity has not been evaluated in MEG recordings up to now.

The purpose of this study was to evaluate the significance of methohexital-activated epileptiform discharges in MEG recordings concerning its value for the lateralization and localization of the primary epileptogenic zone. As a reference method the methohexital-induced activation of spikes and SBS pattern in the ECoG was used. In order to improve statistical conditions, the study was performed in at homogeneous group of patients with temporal lobe epilepsy (TLE).

\section{Methods and materials}

\subsection{Patients}

The clinical. radiological and histopathological data as well as the type of surgery performed and the post-opcrative outcome with respect to seizure control are summarized in Table 1. NII 15 patients (aged 17-44 years, median 37 years) suffered from long-standing temporal lobe epilepsy (duration of epilepsy 11-41 years, median 24 years). Informed consent was obtained from each patient. All patients underwent preoperative evaluation using simultaneous video and ECoG registration of interictal and ictal epileptiform activity. In all but one patient a temporomesial seizure onset was confirmed during the presurgical evaluation. Patient 5 became seizure free after lesionectomy of a temporobasal ganglioglioma without hippocampectomy.

Anticonvulsive medication was maintained at therapeutic levels during both ECoG and MEG recordings.

\subsection{Recording techniques}

During the invasive presurgical evaluation, electrocorticographic recordings were performed using chronically implanted subdural strip electrodes and intrahippocampal depth electrodes (cf. Bonn protocol of presurgical evaluation (Engel, 1993)). In all patients, bilateral temporal subdural strip electrodes with electrode contacts of $2.5 \mathrm{~mm}$ diameter and an intercontact distance of $10-15 \mathrm{~mm}$ were implanted covering the basal, mesiobasal and lateral aspects 
Table 1

Clinical data of the patients

\begin{tabular}{|c|c|c|c|c|c|c|}
\hline $\begin{array}{l}\text { Patient ID/age } \\
\text { (years) initials }\end{array}$ & $\begin{array}{l}\text { Side of } \\
\text { ictial onset }\end{array}$ & $\begin{array}{l}\text { Medication dose }(\mathrm{mg} / \text { day) } \\
\text { serum level }(\mu \mathrm{g} / \mathrm{ml})\end{array}$ & MRI lesion & Surgical procedure & $\begin{array}{l}\text { Histopathological } \\
\text { findings: }\end{array}$ & $\begin{array}{l}\text { Postop follow } \\
\text { up (months)/ } \\
\text { seizure-fres? }\end{array}$ \\
\hline 1/26 B.B. & Right & PHT 350 (19.5), CLB 20 & No p.i. & SAH & AHS & $27 / \mathrm{Yes}$ \\
\hline 2/17 M.A. & Right & CBZ $1800(8.5)$ & No pif. & 2/3 ATLR and IIE & Ileterotopia & $12 / Y c s$ \\
\hline 3/23 K.R. & Right & CBZ $1600(9.9)$ & AHS & $\mathrm{SAH}$ & AHS & $12 / Y \mathrm{Ys}$ \\
\hline 4/38 P.D. & Jefi & CBZ, $1400(9.3)$ & AHS & SAH & AHS and GNH & $24 / \mathrm{Yes}$ \\
\hline $5 / 42$ K.I. & Right & $\mathrm{CB} Z .1800(12.4)$ & Lesion temporo-basal & LE without $\mathrm{HE}$ & GG WHO Gralde I & $24 / Y e s$ \\
\hline $6 / 37$ H.B. & Ieft & CBZ, $1600(10.7)$ & AHS & SAH & AHS & $2+1 Y \mathrm{YC}$ \\
\hline 7/37 H.D. & Right & $C B Z, 1200(10.0)$ & No pif & SAH & AHS & $12 / Y e s$ \\
\hline 8/38 K.D. & Left & CB'L $1200(7.5)$ & AHS & $\mathrm{SAH}$ & AHS & $12 / \mathrm{N}_{0}$ \\
\hline $9 / 34 \mathrm{~V} . \mathrm{H}$ & Right & CB7. $1600(9.9)$ & AHS & SAH & AHS & $12 / Y \times S$ \\
\hline 10/28 S.U. & I.eft & CBZ $1800(9.2)$ & AHS & SAH & AHS & $12 / \mathrm{N}_{0}$ \\
\hline 11/34 H.M. & 1.eft & CBZ $2000(7.1)$ & AHS & $2 / 3$ ATLR and HE & $\mathrm{AHS}$ and $\mathrm{MD}$ & $12 / Y$ Ys \\
\hline 12/43 B.K. & I.eft & CBZ $1600(9.3)$ & AHS & 2/3 ATLR and HE & AHS & 6/Yes \\
\hline 13/37 F.W. & Righı & PHT $350(15.3)$ & AHS & $\mathrm{SAH}$ & AHS & 6/Yen \\
\hline 14/37 D.C. & Right & $\mathrm{CB} \% 1800(9.8)$ & AHS & $\mathrm{SAH}$ & AHS & $6 / Y=s$ \\
\hline 15/44 A.S & Right & $\begin{array}{l}\text { CBZ. } 1650(9.5) \text { : VBT } 2000 \text {, } \\
\text { CL.B. 20) }\end{array}$ & Lesion temporo-mesial & $\mathrm{SAH}$ & GG WHO Grade I & 6 Yes \\
\hline
\end{tabular}

Patient ID: identification number of sach patient; CBZ, carbamazepine, serum concentration of carbamazepine in purentheses, therapeuticil range of CBZ serum concentration: $4-10 \mu \mathrm{g} / \mathrm{ml}$ : PIIT, phenytoin, serum concentration of phenytoin in brackets. therapeutical range of PIIT serum concentration: 5-2() $\mu \mathrm{g} /$ $\mathrm{ml}$; CLB, clobazam, no serum concentration determined; VBT, vigabatrin, no serum concentration determined; no p.t., no pathological findings; AHS. ammonshorn sclerosis: SAH, selective amygdalohippocampectomy; HE, hippocampectomy: 2/3 ATLR, 2/3 anterior temporal lobe resection: LE. lesionectomy: GNH. glioneuronal hamartia; GG. ganglioglioma; MD, migration disorder.

of the temporal lobes. Bilateral intrahippocampal depth electrodes were stereotactically implanted providing 10 cylindrical electrode contacts with a length of $2.5 \mathrm{~mm}$ and an intercontact distance of $4 \mathrm{~mm}$ in the longitudinal axis of the hippocampus on both sides (Fig. 1A). In one patient (No.
15), no intrahippocampal depth electrodes were implanted.

Two to 4 weeks after explantation of the intracranial electrodes the simultaneous recording of MEG and scalp EEG was performed. For scalp EEG recordings (SYNAMPS ${ }^{10}$ : Neuro Scan Inc.) $15 \mathrm{Ag} / \mathrm{AgCl}$-electrodes were

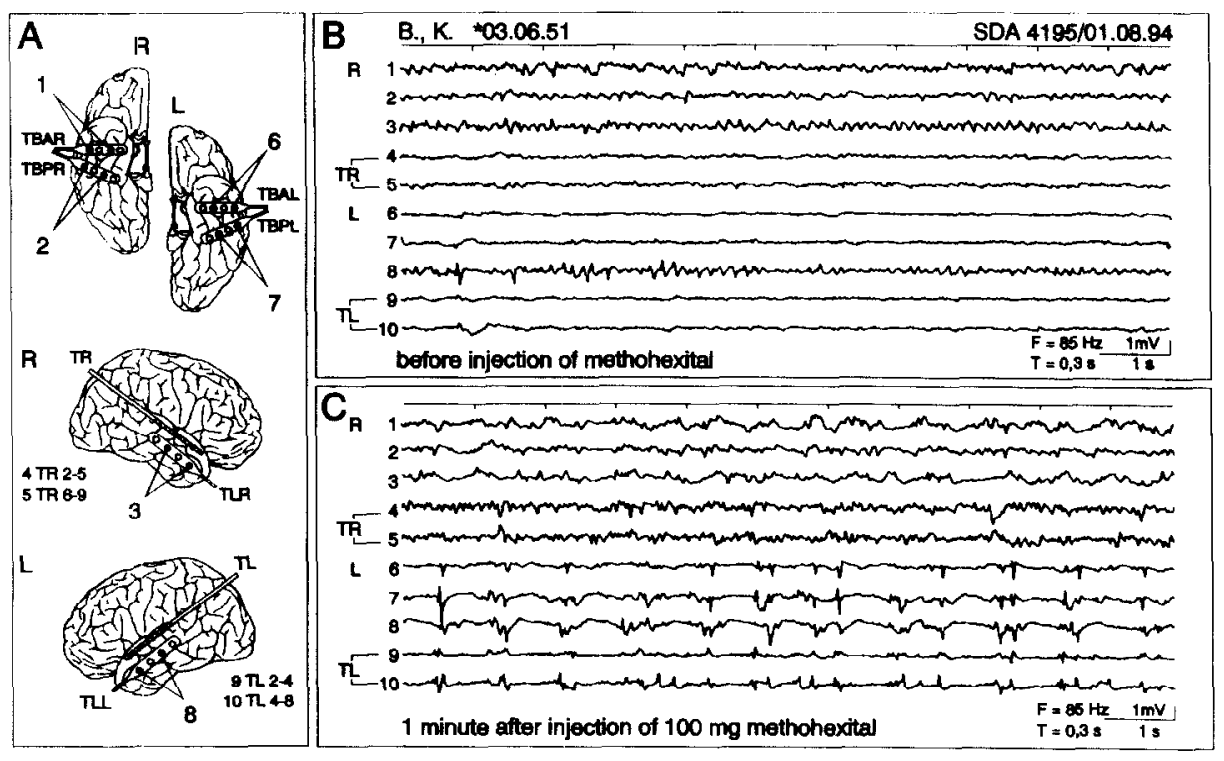

Fig. 1. (A) Scheme of locations of the implanted subdural and depth electrodes. Subdural strip electrodes: TBAR, temporobasal anterior right; TBPR, temporobasal posterior right: TBAL. temporobasal anterior left; TBPL. temporobasal posterior left: TLR, temporolateral right; TLL. temporolateral left. Intrahippocampal depth clectrodes: TR. right depth electrode: TL. left depth electrode: R, right hemisphere: L, left hemisphere. The electrode fizures refer 10 those in the recordings. F. cut of frequency of the low pass filter; T, time constant of the high pass filter. (B,C) Original electrocorticograms of patient 12. (B) Except of a single spike left temporolateral in channel 8. the baseline recordings of the ECoG show no epileptiform activity prior to the administration of methohexital. (C) The ECoG recordings 1 min after intravenous administration of 100 mg methohexital show a marked induction of spikes and spike burst suppression pattern (SBS) left temporobasal (channel 6 and 7 ) and temporolateral (channel 8) as well as in the intrahippocimpal depth electrode (TL: channel 9 and 10 ). 
placed according to the International 10-20 System over the temporal, frontal, central, and parietal brain regions. Additional electrodes were placed at the temporal locations $\mathrm{T} 1$ and T2 according the International 10-10 System (American Electroencephalographic Society, 1991). The nose was chosen as reference, because this location has an equal distance to both temporal lobes and was easy to control during the simultaneous MEG recordings with the patient laying on either side successively. MEG was recorded using a 37 channel neuromagnetometer (Magnes ${ }^{\text {(Iii); }}$ Biomagnetic Technologies Inc.), which consisted of first-order axial gradiometers with $5 \mathrm{~cm}$ baseline. The pickup coils with a diameter of $20 \mathrm{~mm}$ were arranged in an array of concentric circles with a maximal diameter of $12 \mathrm{~cm}$.

The sensor was positioned successively over the hemisphere on either side. The investigators did not know the side of the epileptogenic focus. The sensor was centered 1.5 $\mathrm{cm}$ superior to the electrode locations T3 resp. T4 according the International 10-20 System (Fig. 2). During the MEG recordings patients were lying on their side with their head and body fixed by a vacuum cushion. They were instructed to avoid eye blinks and head movements. MEG recordings were performed in a magnetically shielded room using a sampling rate of $297.6 \mathrm{~Hz}$ and a passband filter of $0.03-$ $100 \mathrm{~Hz}$. The head position relative to sensor pickup coils was measured by a sensor position indicator. Prior to the MEG recordings a MRI scan of each patient was performed (1.5 Tesla; scquences: MPR3D-2_T1_MPR; slicc thickness: $1.5 \mathrm{~mm}$ ). The procedures used to align the different coordinate systems of MEG and MRI recording techniques have been described in detail elsewhere (Pantev et al., 1991).

\subsection{Methohexital-induced activation of epileptiform discharges}

For both the ECoG recordings and the MEG recordings the methohexital application was performed according to the protocol during the presurgical evaluation. The dosage of $100 \mathrm{mg}$ methohexital for each investigation was standardized and not adjusted to the patients weight. During the methohexital administration, anesthetic monitoring and standby was provided in order to counteract complications of the short narcosis. For the ECoG recordings as well as the simultaneous MEG and scalp EEG recordings, a baseline during a period of at least $10 \mathrm{~min}$ was recorded. For the evaluation of spike activation in the ECoG a single methohexital administration was sufficient, whereas for the MEG and EEG recordings methohexital had to be administered twice due to the successive MEG recordings from both hemispheres. In order to allow the recovery of the patients' vigilance to normal levels as well as of the MEG and EEG recordings to return to baseline status the successive recordings were interrupted by a break of 22-89 minutes (mean: $40 \mathrm{~min}$ ).

From ECoG recordings the density of epileptiform poten-
A

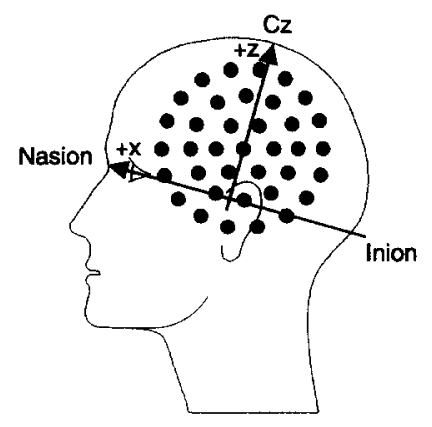

B

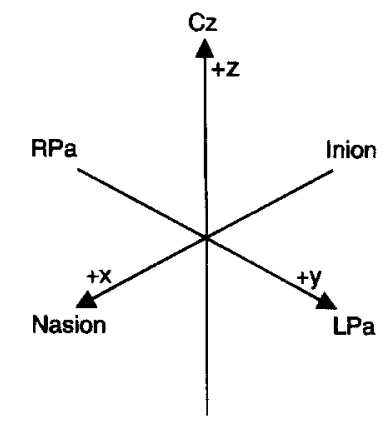

Fig. 2. (A) Scheme of the sensor position and the head frame coordinate system projected onto the patients head. Cz corresponds to the electrode position according the International 10-20 Sytem. (B) Scheme of the head frame coordinate system: the $x$-axis is defined as the line connecting nasion and inion, the $y-a x$ is is defined as the line connecting the left preauricular (LPa) and right preauricular ( $\mathrm{RPa}$ ) point and the z-axis is defind as the line perpendicular to the vertex, indicated by the electrode position $\mathrm{C} z$ according the International 10-20 System.

tials recorded in the temporobasal and temporolateral contacts of the subdural electrodes and in the intrahippocampal contacts of the depth electrodes on either side after methohexital administration was estimated.

Epileptiform potentials in the ECoG were defined as

1. 'Spikes': spikes $(<80 \mathrm{~ms})$ and sharp waves $(80-200$ ms) exceeding the amplitude of the maximal background activity by a factor of 2 .

2. 'Spike burst suppression (SBS) patterns": focal highamplitude spikes followed by short intervals of isoelectric or very slow subdelta background activity.

MEG recordings were evaluated by 2 independent and experienced raters. For the first evaluation, spikes in MEG recordings were accepted only when they were accompanied by a spike in the surface EEG recordings. The first evaluation of the MEG and surface EEG recordings was performed to develop a model of the characteristics of epileptiform discharges recorded in the MEG. In the second evaluation epileptiform discharges recorded with MEG were accepted as a 'spike', if the following conditions were met:

1. The field amplitude had to exceed the maximal amplitude of the background activity by a factor of 2

2. The ascending part of the spike had to rise steeply and reach the peak within $50 \mathrm{~ms}$

3. The latency between ascending and descending part of the spike must not exceed $100 \mathrm{~ms}$

4. The recorded activity should exhibit an approximately dipolar distribution.

\subsection{Comparative analysis of the density of epileptiform potentials in ECoG and $M E G / E E G$ recordings}

In order to evaluate the activation of epileptiform poten- 
tials in the ECoG recordings, the number of spikes/SBS during a period of $3 \mathrm{~min}$ after the methohexital administration were assessed in the temporobasal, temporolateral and intrahippocampal ECoG channels. For the recordings of the MEG and scalp EEG, the spike density during a 3-min period under baseline conditions and during a 3-min period after administration of methohexital were assessed separately. A 'correct' lateralization was assessed if the following conditions were met:

1. The spike density (and SBS density in the case of ECoG recordings) ipsilateral to the primary epilcptogenic area exceeded the contralateral spike (and SBS) density by at least $50 \%$

2. If no spikes were recorded on the contralateral side, the spike (and SBS) density ipsilaterally had to be $\geq 5$ per $3 \mathrm{~min}$.

In the case of a higher spike (and SBS) density contralateral (according to the same criteria as above), a contralateral activation and 'incorrect lateralization' was assessed. If the spike and SBS density ipsi- and contralaterally differed to a lesser degree. the result was rated as a bilateral activation' and in the case of an insufficient increase in densities ( $<50 \%$ or $<5$ spikes/SBS per $3 \mathrm{~min}$ ) as 'no activation'.

\subsection{Analysis of the magnetic field distributions}

Using the raw data of the MEG recordings (i.e., without modeling), the time point corresponding to the spike's peak amplitude with respect to each of the 37 sensors was determined. The sensor where the spike exhibited the highest magnetic field amplitude was identified. Time lags between the peaks of the spike recorded in different sensors with respect to the peak of the spike recorded by the sensor where the spike exhibited its maximal amplitude were calculated. These peak latencies and the topographical distribution of magnetic field extrema of both spontaneous and methohexital-induced spikes were analyzed.

\subsection{Source localization by dipole modeling}

In order to localize the underlying generators of neuronal activity, the single equivalent current dipole (ECD) model based on a moving dipole was applied (Lütkenhöner et al., 1995). Time segments restricted to the ascending part of the spike were selected and entered the subsequent analysis steps. Signal averaging was not performed in order to avoid the reduction of the spatial resolvability by averaging spikes of possibly different origin. For each sampling point of the preselected time segments the magnetic field distribution was calculated. ECD parameters (spatial coordinates and components of the dipole moment) were obtained by minimizing the difference between the measured and the calculated field distribution. The resulting spatial coordi- nates of the estimated ECD were considered to represent locations of the underlying source if the root mean square (RMS) of the measured magnetic signal and the goodnessof-fit (GOF) of the minimization procedure simultaneously exceeded certain empirically defined threshold values (GOF $\geq 98 \%$, RMS $\geq 600 \mathrm{fT}$ ). These threshold values were selected in order to guarantee that only equivalent current dipoles corresponding to the spikes' absolute maxima were selected.

Additionally, source localizations were calculated using a spatio-temporal dipole model based on a single rotating dipole (Lütkenhöner et al., 1991: Lütkenhöner, 1991: Scherg, 1994; Scherg and Ebersole. 1994. Lütkenhöner et al., 1995). As for the moving dipole. time segments were restricted to the ascending part of the spike. In order to estimate the ECD parameter 10 randomized starting values of the fit procedure were used. The starting values were characterized by a source location within a predefined volume and a predefined orientation. Only stable solutions. defined as being independent on starting values, entered the subsequent analysis steps. In case of several stable, but different ECD solutions the most frequent one was assumed to represent the location of the source generating the epileptiform discharge.

For both dipole models the source localizations were calculated based on the assumption of a spherical head model using a homogeneous sphere that best fitted the patients" head underneath the sensor array. The coordinates of the dipole localization were determined in a head frame coordinate system adjusted to sensor position (Fig. 2). After source localization, a source location density analysis was performed in order to find correlations between the calculated ECD locations obtained from the applied models and the primary epileptogenic area determined during the presurgical evaluation. For this purpose. the volume of each patient's head was subdivided into equidistant cubicles of $64 \mathrm{~cm}^{3}, 8 \mathrm{~cm}^{3}$ and $1 \mathrm{~cm}^{3}$. For each size of the cubicles. the number of calculated source locations within a cubicle was estimated. Cubicles comprising at least 3 dipole source locations were projected onto the patient's MRI scans in order 10 allow neuro-anatomical correlations.

\subsection{Statistical analvsis}

The Wilcoxon signed-ranks test for paired samples was applied to compare spike densities of the successive baseline MEG and EEG recordings as well as before and after methohexital administration and to compare spike/SBS-densities ipsi- and contralateral to the side of the epileptogenic focus in MEG. EEG and ECoG recordings.

Correlations between spike and SBS densities in the ECoG and spike density in the MEG and EEG were evaluated using the bivariate Spearman's coefficient. A significant correlation was accepted if $P<0.05$ was obtained. 


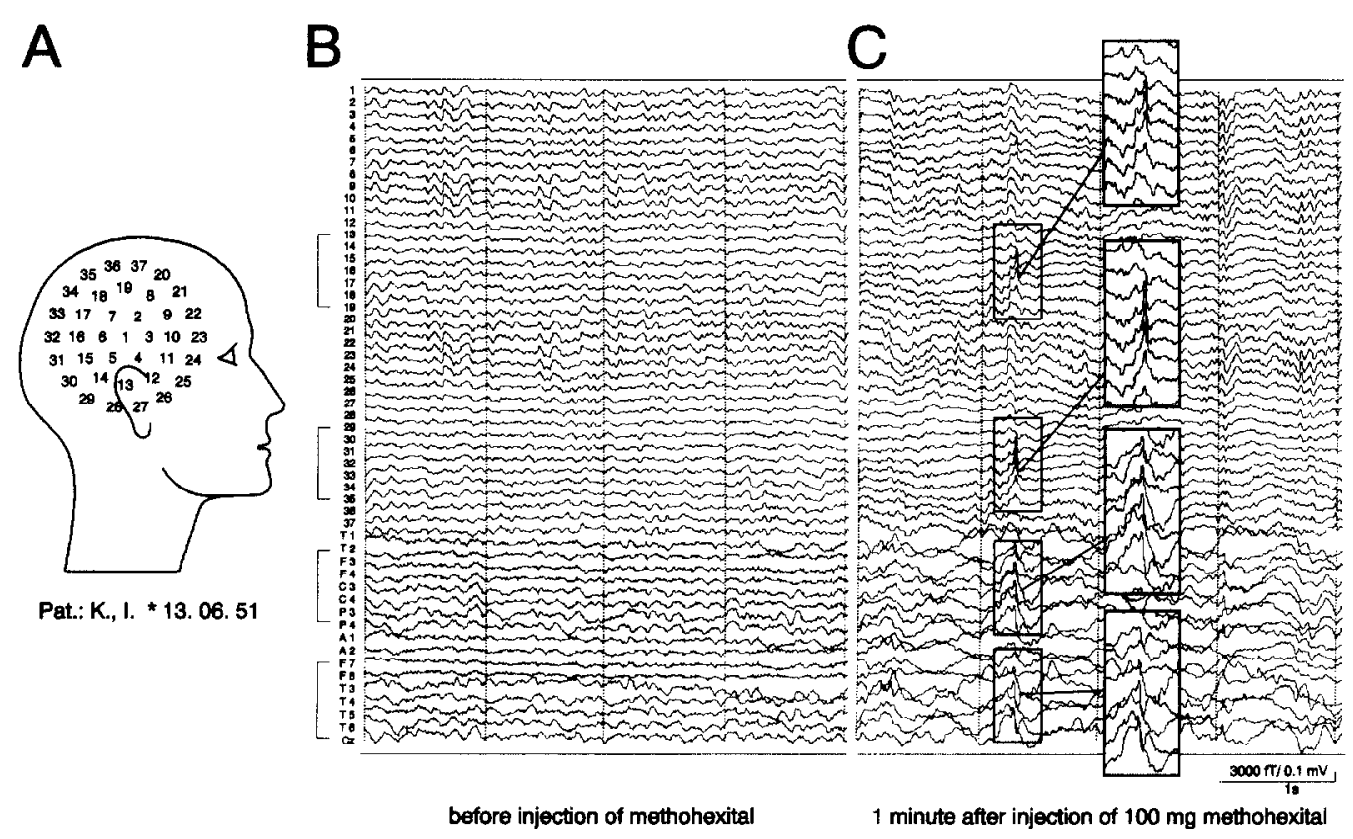

Fig. 3. MEG recorded over the right hemisphere and scalp EEG recordings of patient 5. Channels $1-37$ refer to the magnetic sensors, channel $\mathrm{Tl}$ to $\mathrm{Cz}$ to the scalp EEG electrodes according the International 10-20 System. (A) Scheme of MEG sensor positions. (B) The baseline recordings show no spontaneous spikes. (C) After methohexital application the spike density increases in the MEG recordings as well as in the scalp EEG recordings.

\section{Results}

\subsection{Clinical effects of methohexital}

No adverse effects during or after the short narcosis were noticed by either the patient or the investigators. Patients fell asleep 30-60 s after application of methohexital, recovered quickly within $10 \mathrm{~min}$ and had no clinical excitatory effects, nausea or vomiting. No seizure was elicited.

\subsection{Analysis of the density of spontaneous and methohexital-induced epileptiform potentials in ECoG, $M E G$, and $E E G$ recordings}

An example of methohexital evoked epileptiform discharges in the ECoG recordings is presented in Fig. 1B and $C$ showing a marked activation of spikes and SBS in the intrahippocampal, temporobasal and temporolateral recordings ipsilateral to the epileptogenic focus.

Prior to the first and second methohexital application the MEG and EEG recordings showed no differences in baseline spike densities. After methohexital administration a significant increase in spike density was observed. This spike induction was more pronounced ipsilateral to the epileptogenic focus than over the contralateral hemisphere (Figs. 1B,C, 5). In 7 of the 9 patients who exhibited spontaneous spikes during the baseline MEG recordings, the field amplitudes of the methohexital-induced spikes were higher as compared to the field amplitudes of the spontaneously occurring spikes. Automatic spike detection was not assessed because a discrimination between spikes and drug-induced beta-activity was difficult to perform in 5 of the 15 patients. Ilowever, the scalp EEG recordings often proved helpful, although a spike detectable in the MEG was not always accompanied by a spike in the EEG recordings. $\ln 7$ of the 15 patients spikes in the MEG recordings without accompanying spike in the EEG recordings occurred. In these 7 patients on average $37 \%$ of the MEG recorded spikes (10 68\%) were not accompanied by a spike in the EEG recordings. In most patients, visual detection of spikes in the MEG recordings was easy to perform (Figs. 3,4).

\subsection{Lateralizing significance of methohexital induced epileptiform discharges}

The number of spikes and SBS detected during the period of 3 min after methohexital application in ECoG, MEG, and scalp EEG ipsilateral to the epileptogenic focus are presented in Table 2.

3.2.1.1. ECOG recordings. The lateralizing significance of methohexital induced epileptiform discharges in the FCoG differed between the 3 different temporal brain regions as well as between spikes and SBS. Comparison between ipsiand contralateral spike densities showed statistically significant differences $(P<0.01)$ for the temporobasal as well as for the intrahippocampal depth recordings. Differences of SBS densities between ipsi- and contralateral recordings did not reach statistical significance nor did the spike resp. SBS densities recorded in the temporolateral contacts.

3.2.1.2. MEG recordings. In contrast to the spontaneously occurring spikes, the difference between the methohexital- 


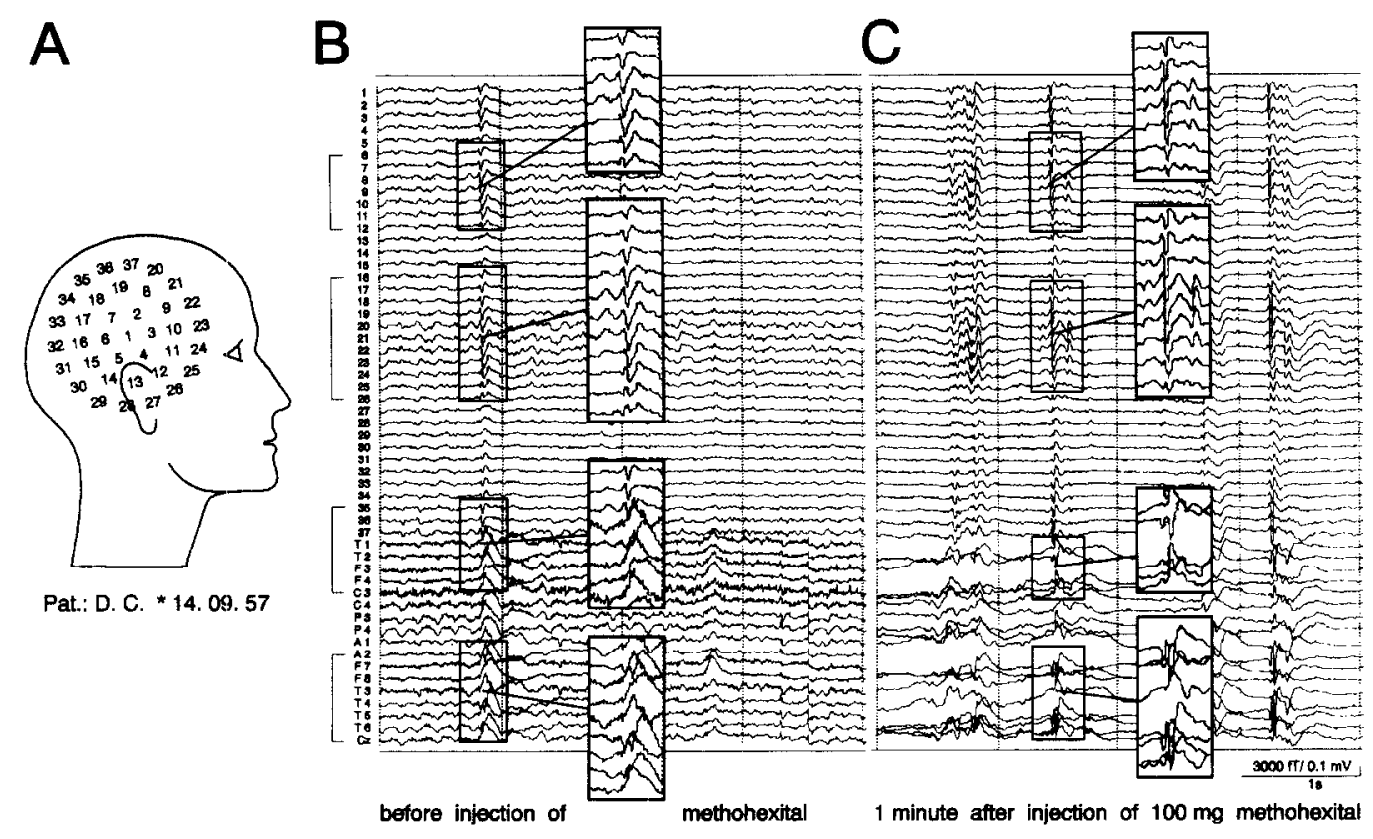

Fig. 4. Same as Fig. 3, but recordings of patient 14 (right hemisphere). (A) Scheme of MEG sensor positions. (B) The baseline recordings show a typical interictal period with a low spike density (one spike at the end of the first second of the presented recording period). (C): After methohexital application spike density and amplitude increases in the MEG recordings as well as in the scalp EEG recordings from the right hemisphere. The spikes are followed by a suppression of brain activity similar to the SBS observed in the ECoG recordings.

induced spike density in the MEG recordings ipsi- and contralateral was statistically significant $(P<0.05)$ (Fig. 5).

The lateralization power of the spike density of methohexital-induced spikes in the MEG recordings concerning the side of the primary epileptogenic area proved to be close to that of methohexital-induced spikes in the ECoG recordings in this selected group of patients with TLE. As summarized in Tables 3 and 4 , the spike densities of methohexital-induced spikes and SBS in the temporobasal record-

Table 2

Comparison of individual ipsilateral spike densities

\begin{tabular}{|c|c|c|c|c|c|c|c|c|c|c|}
\hline \multirow{3}{*}{$\begin{array}{l}\text { Patient } \\
\text { II) }\end{array}$} & \multicolumn{2}{|c|}{ MEG spike density } & \multicolumn{2}{|c|}{ EEG spike density } & \multicolumn{6}{|c|}{ ECoG density of spikes and spike-burst-suppression } \\
\hline & \multirow[t]{2}{*}{ Spontaneous } & \multirow[t]{2}{*}{ Induced } & \multirow[t]{2}{*}{ Spontaneous } & \multirow[t]{2}{*}{ Induced } & \multicolumn{2}{|c|}{ Hippocampal } & \multicolumn{2}{|c|}{ Temporobasal } & \multicolumn{2}{|c|}{ Temporolateral } \\
\hline & & & & & Spikes & SBS & Spikes & SBS & Spikes & SBS \\
\hline 1 & 0 & 0 & 0 & 0 & 15 & 102 & 33 & 0 & 0 & 0 \\
\hline 2 & 11 & 25 & 10 & 23 & 4 & 97 & 0 & 1.15 & $y$ & it \\
\hline 3 & 7 & 19 & 14 & 5 & 290 & 0 & 144 & 0 & 12 & 0 \\
\hline 4 & 5 & 21 & 6 & 16 & 197 & 0 & 184 & 0 & 0 & 0 \\
\hline 5 & 2 & 90 & 3 & 53 & 121 & 1.30 & 184 & 44 & 21 & $\gamma$ \\
\hline 6 & 1 & 8 & 2 & 28 & 1.37 & 0 & 73 & 0 & 0 & 0 \\
\hline 7 & 9 & 10 & 6 & 18 & 142 & 18 & 212 & 23 & 21 & 10) \\
\hline 8 & 0 & 0 & 0 & 0 & 36 & 0 & 34 & 0 & () & 0 \\
\hline 9 & 0 & 11 & 0 & 9 & 92 & 197 & 191 & 15 & t & 2 \\
\hline 10 & () & $2:$ & 0 & 30 & 37 & 6 & 53 & 15 & 6 & 2 \\
\hline 11 & 0 & 0 & 1 & 7 & 73 & 6 & 0 & 0 & 0 & 0 \\
\hline 12 & 1) & 96 & 23 & $16 ?$ & 152 & 140 & 161 & 149 & 73 & 106 \\
\hline 1.3 & 0 & 2 & 0 & 1 & 39 & 23 & 25 & 51 & 0 & () \\
\hline 14 & 17 & 210 & 19 & 217 & 72 & 78 & 102 & 129 & 49 & 37 \\
\hline 15 & 7 & 27 & 3 & 44 & - & - & 104 & 0 & $x$ & 77 \\
\hline Median & 2 & 19 & 3 & 18 & 83 & 21 & 39 & 15 & 6 & 2 \\
\hline
\end{tabular}

Figures refer to number of spikes resp. spike burst suppression (SBS) during the period of $3 \mathrm{~min}$ (spike resp. SBS density). The MEG- and scalp EEG recordings were evaluated during baseline (spontaneous spike density) and after methohexital administration (induced spikes). The ECoG recordings were evaluated after methohexital administration with respect to spikes and SBS in the three temporal regions (intrahippocampal. temporobasal and temporolateral). 


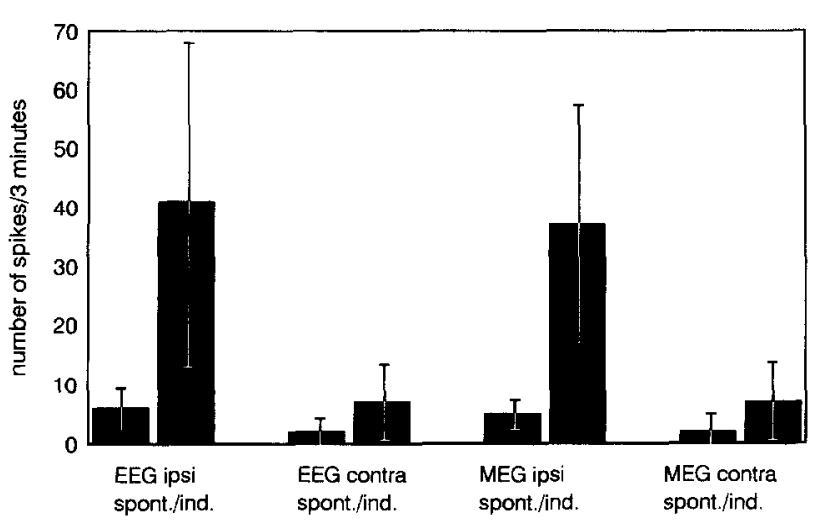

Fig. 5. Bars indicate the mean spike density in the EEG recordings ipsilateral (EEG ipsi) and contralateral (EEG contra) as well as in the MEG recordings ipsilateral (MEG ipsi) and contralateral (MEG contra) to the primary epileptogenic focus. The error bars indicate the standard deviation. (spont.. spontaneous spike density. ind., induced spike density after methohexital application). Mean spike densities in EEG and MEG recordings increase significantly after methohexital administration compared to the density of spontaneous spikes ipsilateral $(P<0.001)$ and contralateral $(P<0.05)$ to the epileptogenic focus.

ings allowed to correctly lateralize the primary epileptogenic area in 13 of 15 patients. Intrahippocampal and temporolateral epileptiform potentials had a poorer lateralizing value. The spike density of methohexital-induced spikes in the MEG recordings allowed a correct lateralization in 9 of the 15 patients. Spontaneously occurring spikes were less frequent and allowed a correct lateralization only in 5 of the 15 patients. Spike density analyses in the EEG recordings provided similar results as were obtained from the MEG recordings.

\subsubsection{Correlation between spike densities in $M E G$} recordings and spike/SBS densities in the $E C O G$ recordings

Three patients did not exhibit spikes in the MEG recordings (patients 1, 8 and 11). In the ECoG recordings of these 3 patients epileptiform discharges were restricted to the temporomesial region with spikes recorded in the intrahippocampal contacts. Patient 1 and 8 showed additional spike activation in the adjacent temporobasal contacts of the subdural electrodes, but none of the 3 patients exhibited any spikes or SBS in the temporolateral contacts.

Correlations between the spike density in the MEG recordings and the density of epileptiform discharges in the ECoG recordings varied depending on the brain region (intrahippocampal, temporobasal or temporolateral) as well as on the type of epileptiform discharges (spike or SBS) in the ECoG recordings. A significant positive correlation was obtained for the temporobasal SBS density with the spike density in the MEG recordings ipsilateral to the focus ( $P<$ $0.001)$. Furthermore, the correlation between the temporolateral spike density and the ipsilateral MEG spike density was statistically significant $(P<0.001)$. In contrast, no significant correlations were obtained between the intrahippocampal spike or SBS density and the MEG spike density.

Analyzing the amplitudes of spikes or SBS, no significant correlation between the amplitudes of epileptiform potentials in the ECoG recordings and the spike density or the field amplitudes of spikes in the MEG recordings were observed.

\subsection{Analvsis of the peak latencies of the spikes recorded in the $M E G$}

In order to determine whether a single generator activation or a successive activation of different generators took place, we measured the differences between time points where a single spike exhibits its highest magnetic field amplitude at different sensor locations in each patient. We observed a mean difference of the peak latencies of $9 \pm 5$ $\mathrm{ms}$ for spontaneous spikes and $6 \pm 3 \mathrm{~ms}$ for methohexitalinduced spikes. The difference between spontaneous and methohexital-induced spikes was not statistically significant. In a single spike an anterior-posterior shift in the peak latencies could be observed (Fig. 6). With regard to all patients, no preferred direction (either anterior-posterior or superior-inferior) was observed.

\subsection{Analysis of the magnetic field distribution}

Analyses of the magnetic field distribution in the temporal evolution of the spikes revealed a high spatial variability of the field extrema, even at the time when the spike reached its apex. Furthermore, field extrema were predominantly located at the margin of the area covered by the sensor as shown in Fig. 6.

Table 3

Classification with respect to the primary epileptogenic area based on the ECoG data

\begin{tabular}{|c|c|c|c|c|c|c|c|c|c|c|c|c|}
\hline \multirow[t]{2}{*}{$\mathrm{ECOG}$} & \multicolumn{3}{|l|}{ Correct } & \multicolumn{3}{|c|}{ Contralateral } & \multicolumn{3}{|c|}{ Bilateral } & \multicolumn{3}{|c|}{ No Activation } \\
\hline & Spikes & SBS & $\begin{array}{l}\text { Spikes } \\
+ \text { SBS }\end{array}$ & Spikes & SBS & $\begin{array}{l}\text { Spikes } \\
+ \text { SBS }\end{array}$ & Spikes & SBS & $\begin{array}{l}\text { Spikes } \\
+ \text { SBS }\end{array}$ & Spikes & SBS & $\begin{array}{l}\text { Spikes } \\
+ \text { SBS }\end{array}$ \\
\hline Intrahippocampal & 12 & 8 & 9 & I & 5 & 4 & 1 & 0 & 1 & 1 & 2 & 1 \\
\hline Temporo-basal & 12 & 7 & 13 & 1 & 2 & 0 & 1 & 1 & 1 & 1 & 5 & 1 \\
\hline Temporo-lateral & 7 & 7 & 8 & 1 & $\mathrm{l}$ & l & 1 & 0 & 1 & 6 & 7 & 5 \\
\hline
\end{tabular}

Lateralization of the epileptogenic area based on spike and SBS densities in the ECoG recordings after methohexital application. Figures refer to numbers of patients in each classification group. 
Table 4

Classification with respect to the primary epileptogenic area based on the MEG and EEG data

\begin{tabular}{|c|c|c|c|c|c|c|c|c|}
\hline & \multicolumn{2}{|l|}{ Correct } & \multicolumn{2}{|l|}{ Contralateral } & \multicolumn{2}{|l|}{ Bilateral } & \multicolumn{2}{|l|}{ No activation } \\
\hline & Spontaneous & Induced & Spontaneous & Induced & Spontaneous & Induced & Spontaneous & Induced \\
\hline MEG & 5 & 9 & 2 & I & 0 & 2 & 8 & 3 \\
\hline EEG & 6 & 10 & 1 & 1 & 0 & 1 & 8 & 3 \\
\hline
\end{tabular}

Lateralization of the epileptogenic area based on the spike densities in the MEG and EEG recordings hefore and after methohexital application. Figures refer to numbers of patients in each classification group.

\subsection{Dipole localization of methohexital-induced and spontaneously occurring spikes in the $M E G$ recordings}

The results of the dipole source localizations on the basis of the spatio-temporal rotating dipole model did not differ substantially from the results obtained with the moving dipole model. With increasing size of the cubicles the number of dipole source locations within a cubicle increased in all patients of the sample. In cubicles of $8 \mathrm{~cm}^{3}$ the density of dipole source locations was iwice as high as in cubicles of 1 $\mathrm{cm}^{3}$ (range: $1-3.3$ ). In cubicles of $64 \mathrm{~cm}^{3}$ the density of source locations was 2.1 folded higher than in cubicles of $8 \mathrm{~cm}^{3}$ (range: $1.3-2.89$ ). In the following the results of the classification based on a cubicle size of $8 \mathrm{~cm}^{3}$ is going to be presented. because this volume best resembles the volume of the amygdala and the anterior part of the hippocampus.

Twelve of the 15 patients exhibited spikes in the MEG recordings after methohexital injection. According to the source location density analysis, in 6 of the 12 patients more than 3 dipole source locations were found within cubi cles of $8 \mathrm{~cm}^{3}$. After projecting these cubicles into the brain MRI scans of each patient, 4 groups of patients could be distinguished.

For the first group (patients 5, 12 and 14) localizing results were obtained that correspond to the epiletogenic area defined according the results of the presurgical evaluation. The MRI projections of the cubicles were located within the parahippocampal gyrus or the adjacent temporal cortex as illustrated in Fig. 7. These patients exhibited a marked activation of spikes in the MEG after methohexital of 90,96 and 216 spikes $/ 3$ min in the MEG recordings, and a high source location density per cubicle was obtained in these patients.

The second group assembled patients $(6,13$ and 15) with poor localizing results. These patients exhibited only a low spike activation in the MEG after methohexital administration and the source location density per cubicle was correspondingly low. The source locations projected into the frontal subcortical layer in patient 6 (one cubicle with 8 dipole source locations), into the parietal cortex and into the parahippocampal gyrus in patient 1.3 as presented in Fig. 8 (2 cubicles with 5 and 3 dipole source locations) and onto the temporolateral skull in patient 15 (one cubicle with 3 dipole source locations).

The third group consisted of patients $(2-4,7,9$ and 10) with a moderate spike activation in the MEG recordings, but without consistent results in the source density analysis. In these patients, a spatial density of at least 3 dipole solutions per cubicle was not achieved. Consequently, no projection of cubicles into the MRI scans was possible in these patients.

As already mentioned, the fourth group (patients I, 8 and 11) did not exhibit spikes in the MEG recordings. Therefore. no source localization was possible in these patients. In this

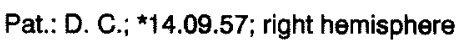

A

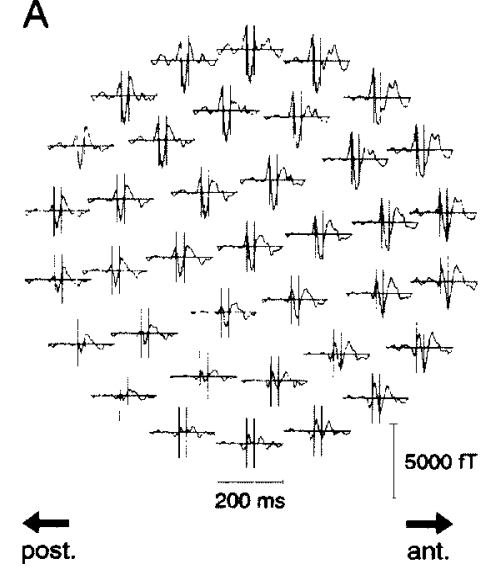

B
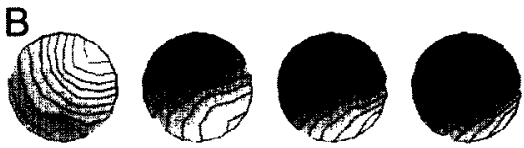

$2309.00 \mathrm{TT}$

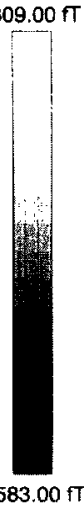

Fig. 6. Spatial distribution of a single methohexital-induced spike of patient 14 projected onto the sensor layout (part A) and the corresponding magnetic tield distributions (part B) calculated for the preselected time segment indicated by the vertical lines in part $\wedge$. Ant., anterior. Post.. posterior. Time interval between each magnetic tield distribution map is $6.7 \mathrm{~ms}$. During the time course of the spike, the magnetic field distribution exhibits a considerable spatial variahility. Even during the peak of the spike, the extrema of the magnetic field are located at the margin or outside the area covered by the sensor array. 


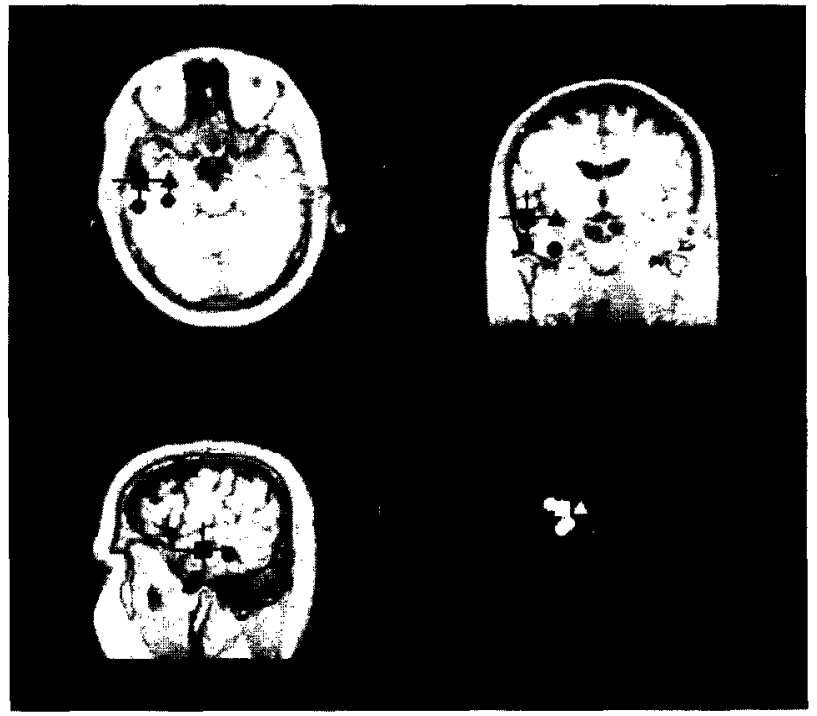

Fig. 7. Projections of the center of the $8 \mathrm{~cm}^{3}$ cubicles including at least 3 dipole solutions of patient 14 onto his MRI scans. The cubicles are indicated by the following symbols: $\square$ (35 solutions). $\Delta$ (29 solutions), $O 113$. 14 or 15 solutions). Projections of the cubicles onto the axial plane (upper lefi), onto the coronal plane (upper right), onto the sagittal plane (lower left) and unto the head lanne coordinate system.

group of patients, no spikes or SBS were detected in the temporolateral contacts of the subdural electrodes.

With respect to the activation of epileptiform activity in the ECoG, there were no clear differences between patients with good and poor localizing results. Patients with good results of the MEG source localizations all exhibited a marked activation of spikes and SBS in the intrahippocampal, temporobasal and temporolateral contacts. But in the group of patients with poor or missing localizing results, the activation in the ECoG was low in about half of the patients. but well pronounced in the other half. No consistent relations between the density of spikes and SBS in the different temporal regions (intrahippocampal, temporobasal and temporolateral) recorded in the $\mathrm{ECoG}$ and depth recordings on the one hand and the source localizations of the MEG recorded spikes on the other were obtained.

During the measurement period of 3 min the spike density of spontaneous spikes was significantly lower (Fig. 5) than after methohexital application. Analyzing the source localizations of spontaneous spikes, in none of the patients the criterion of a spatial density of at least 3 dipole solutions restricted to a cubicle of $8 \mathrm{~cm}^{-4}$ was met. Therefore, no projection of cubicles into the MRI scans for the spontaneous spikes was possible.

\section{Discussion}

The purpose of this study was to evaluate the possibilities and limitations of non-invasive simultaneous multi-channel MEG and scalp EEG recordings concerning the lateralization and localization of the epileptogenic zone. The value of spontaneous and drug-induced epileptiform activity was analyzed and compared to the results of ECoG and depth recordings of the same patients. The study was performed in a clearly defined group of patients with unilateral temporal lobe epilepsy (TLE) confirmed by the invasive presurgical evaluation as well as by the histopathological findings and the postsurgical outcome. The present study demonstrates that methohexital administration is a simple and fast method to increase the spike density in the MEG recordings and to shorten the registration time. The diminution of movement artifacts during the short narcosis is another advantage of the methohexital application. For ECoG recordings it has been shown in several investigations that methohexital application increases the density of epileptiform discharges of high localizing value (Bickford et al.. 1953; Musella et al., 1969; Ford et al., 1982; Aasley et al., 1989; Hufnagel et al., 1992). The lateralizing and localizing results of methohexital-induced epileptiform discharges are reproducible and independent from serum levels of anticonvulsive drugs (Kowalik et al., 1997). In most patients investigated in our study anticonvulsive medication consisted of a carbamazepine monotherapy within the therapeutical range. Therefore, differences in drug interactions can be neglected in these patients. In those 2 of our patients receiving clobazam, effects on the spike activation can not be excluded. However, no systematic influence was observed since in patient 1 no spikes were detected in the MEG recordings, whereas patient 15 exhibited a clear spike induction.

The comparison between ECoG and MEG recordings confirmed that the value of methohexital-induced spikes in non-invasive MEG recordings concerning the lateralization of the epileptogenic area is comparable to that of methohexital-induced spikes in invasive $\mathrm{ECOG}$ recordings. Furthermore, methohexital-induced spikes in the MEG recordings are superior to spontaneous spikes concerning

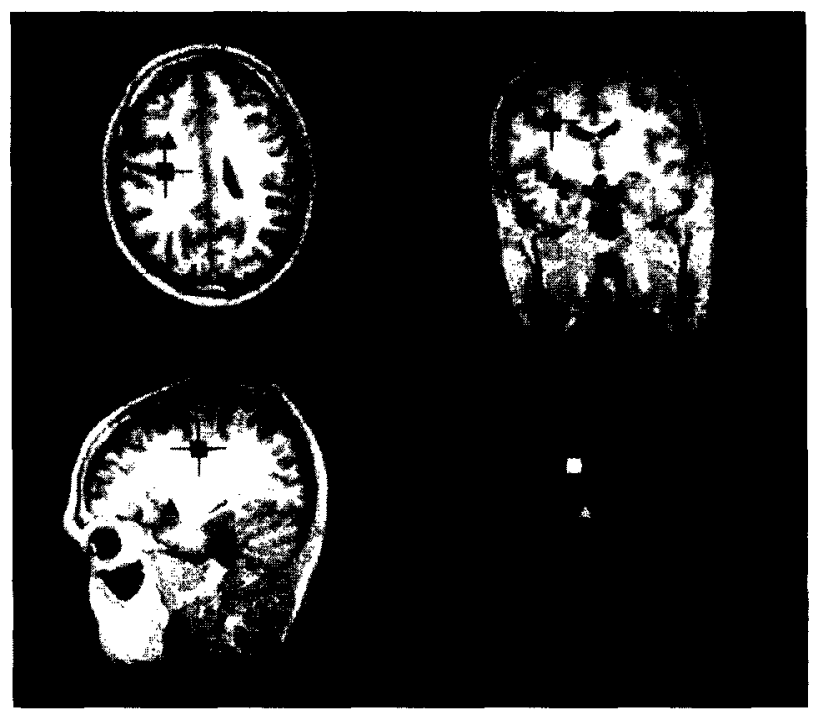

Fig. 8. Same as Fig. 7. but for patient 13. $\square$ (.5 solutions). $\triangle 1.3$ solutions). 
their lateralizing significance. since only few patients exhibited enough spontaneous spikes within comparable registration times.

However, a reliable localization of the epileptogenic zone remains still difficult. The results of the methohexitalinduced epileptiform discharges in MEG recordings indicate that, under the experimental conditions and assumptions of this study, the MEG is capable to detect epileptiform discharges elicted in the more lateral parts of the temporal lobe. whereas the spikes and SBS generated in temporomesial structures seem to escape the MEG registration. This can be concluded by comparing the MEG spike density to the spike and SBS density in the ECoG recordings.

For clinical purposes of an exact localization and delineation of the primary epileptogic zone, a source locali-ation within a volume of $1 \mathrm{~cm}^{3}$ would be desirable. The present study showed that consistent localizatory results can be achieved for cubicles of $8 \mathrm{~cm}^{3}$, corresponding to a volume of the amygdala and the anterior part of the hippocampus, in 3 of the 15 patients. Source estimations of spikes recorded in the MEG resulted in localizations within the parahippocampal gyrus and the adjacent temporal cortex in only 3 patients, whereas in another 3 patients widespread localizations into frontal and parietal regions were obtained. For the remaining 9 patients no consistent source locations were obtained. Cubicles of $64 \mathrm{~cm}^{3}$. corresponding to the volume of the whole temporal lobe, do not provide sufficiently precise localizatory information that is required for epilepsy surgery.

The results reported here can only be discussed on the basis of previously reported simulation studies (Gharib et al. 1995) and analyses of spontaneous interictal spike recordings in the MEG. hecause to our knowledge no studies concerning drug-induced epileptiform activity in $\mathrm{MEG}$ recordings have heen described before. MEG recordings of interictal spontaneous spike activity in patients with focal epilepsy have been found to be of limited value for the localization of deep sources in the temporal lobe. In order to compare the localizing value of scalp FFG, FCoG, and MEG. Nakasato et al. (1994) evaluated 5 patients with neocortical epilepsy and one patient with temporomesial epilepsy using a 7-channel magnetometer. In 3 of 6 patients good localizing results with MEG were obtained. but in the patient with temporomesial epilepsy no source localization was possible, because no spikes were detected in the MEG recordings. Tiihonen et al. (1990) presented a study in 2 subjects with TL.E using a 7-channel gradiometer. They were able to localize sources into the lateral aspects of the temporal lohe. whereas the results of the preoperative evaluation suggested a temporomesial epileptogenic area. A recent study on 50 patients with focal epilepsy showed that $\mathrm{MEG}$ recordings using a 37-channel first-order axial gradiometer provided good localizing results for patients with convexity foci, whereas source localizations in patients with orbitofrontal or deep foci did not agree with the results of the presurgical evaluation (Smith et al., 1995). These studies indicate that even with a 37-channel system the source localization of spontaneous interictal epileptiform activity generated in deep structures as the temporomesial region is still difficult.

The low localizing value of MEG recordings concerning deep sources may have different reasons. In order to solve the inverse problem. the algorithms used to localize neuromagnetic sources are based on several assumptions concerning measurement conditions. source contiguration. and head models.

\subsection{Measurement conditions}

The fact that the extrema of the magnetic tields were predominantly located at the margins of the area covered by the sensor represents one main reason for the limited results of the source localization procedure. The sensor array of 37-channel gradiometer used in this study had a maximal distance of sensor points of $12 \mathrm{~cm}$ that might be too small to correctly localize deep sources (Ahonen et al.. 1993). Thus, whole-head MEG-systems may be better suitable to solve this problem. Besides the configuration of the sensor array, the baseline of the gradiometer influences the power to record magnetic fields of deep sources. The gradiometer used in our study had a baseline of $5 \mathrm{~cm}$, which does not yield a very good signal-to-noise ratio ( $\mathrm{S} / \mathrm{N}$ ratio) in the case of decp sources (i.c. $6-7 \mathrm{~cm}$ ). The $\mathrm{S} / \mathrm{N}$ ratio is one of the most important parameters that might influence the localization power of the MEG. With increasing depth of the source, the signal intensity decreases. i.e. the $S / N$ ratio decreases (Ogura and Sekihara, 1993; Supek and Aine. 1993: Cohen el al., 1990). By performing MEG recordings: in a magnetically shielded room, the external noise has been reduced as much as possible in this study. However, the noise caused by the brain activity itself cannot be eliminated. Signal averaging can improve the $\mathrm{S} / \mathrm{N}$ ratio. However, averaging of spikes has not been performed in this study in order to avoid the risk of averaging ativity generated in different brain areas.

\subsection{Sonre configuration}

Little is known about the source configuration corresponding to epileptiform activity in focal epilepsies. Only the depth of the source can be estimated because our study is based on a group of patients with temporal lobe epilepsy (TLE), in all but one patient with a temporomesial seizure onset confirmed by the presurgical epilepsy evaluation including invasive video monitoring. According to the results of the previously performed ECoG recordings of the methohexital-induced epileptiform potentials, the spatial distribution of the generators of the epileptiform activity could be demonstrated. Yet, dipole moment and orientation are not known. This study was based on the assumption that the location of the generators of epilentiform activity in the 
ECoG and the MEG recordings remains invariant. It has been shown previously that the methohexital activation provides reproducible results with respect to localization and lateralization of the primary epileptogenic area (Kowalik et al., 1997). Nevertheless, in order to be sure about the origin of a spike or SBS, it would be necessary to perform simultaneous recordings of the ECoG and MEG

The implications of drug-induced epileptiform activity compared to spontaneous spike activity for the source configuration are not known. Difficulties in determining the exact source localization even in our study on a highly uniform group of patients may be due to the fact that spontaneous spikes as well as methohexital-induced spikes and SBS are generated in rather extended brain areas. The ECoG recordings showed that methohexital-induced spikes and SBS can be recorded not only in the temporomesial structures, but over the temporobasal and temporolateral cortex as well. According to Wyler et al. (1987), Hufnagel et al. (1992), and Kowalik et al. (1997), the methohexitalinduced spikes and SBS are of good localizing value concerning the primary epileptogenic zone and superior to the localizing value of interictal spikes, which reflect the irritative zone (Lüders and Awad, 1991). Methohexital causes not only a quantitative increase in epileptiform pattern, but also a qualitative change with induction of high-amplitude SBS in the ECoG recordings (Hufnagel et al., 1992). With respect to our results, the localizing value of methohexitalinduced spikes recorded with the MEG is superior compared to spontaneous interictal spikes due to the increased spike density and a higher specificity with respect to the epileptogenic area.

The solution of the inverse problem is based on several assumptions concerning the source configuration, such as the number of active sources and their spatial separation. For our patients with TLE we would have expected source localizations in a depth of $6-7 \mathrm{~cm}$. The limits of source resolutions for a multiple dipole model grow with increasing depth (Supek and Aine, 1993; Lütkenhöner et al., 1991). Approximately, the distance at which 2 sources can be resolved using magnetic field measurements is comparable to the distance between source and sensor (Tan et al., 1990). In accordance with these theoretical assumptions, single dipole models are adequate models for the source localization procedure. Simulation studies showed that the resolving power in case of a spatio-temporal dipole model based on a rotating dipole resp. a fixed dipole is only slightly superior compared to a moving dipole model (Lütkenhöner et al., 1991). In accordance with these results, the results of the present study showed that the source locations obtained with the spatio-temporal dipole model based on a rotating dipole did not lead to different localizing results compared to the results obtained by the single ECD based on a moving dipole. Nevertheless, the widespread activation of epileptiform potentials in the ECoG recordings after methohexital and the differences in peak latencies of the spike maxima indicate that single dipole models can not be regarded as adequate models to describe the underlying source. Whether distributed source models (Nunez, 1986; de Munck et al.. 1992; Supek and Aine, 1993) are more suitable to describe the source has to remain open. Differences in peak latencies of the maxima of a single spike have already been observed in animal models of focal epilepsies (Petsche et al., 1969; Elger and Speckman, 1980), suggesting a successive activation of different generators.

According to Alarcon et al. (1994), human interictal spike activity recorded in the scalp EEG does not arise from volume conduction from deep structures, but is generated in the underlying neocortex. Within milliseconds interictal activity can propagate and successively activate large cortical areas due to 3 possible mechanisms. Fast association fibers can activate remote cortical areas either directly or by triggering local activity. Furthermore, propagation can take place along the neocortex. Thus, it was concluded that due to these propagation mechanisms the assumption of a single dipole or multiple-dipole model is not adequate to describe interictal activity at least for EEG recordings and that the analysis of propagation mechanisms may be more fruitful (Alarcon et al., 1994). These results confirm our hypothesis that one reason for the limited localizing value of MEG recorded spikes may be the propagation of epileptiform activity to the neocortex near the MEG recording site. Thus, the epileptiforn activity generated in the temporolateral cortex may mask the activity generated in deeper structures. Further studies are necessary to answer the yuestion whether MEG systems with an improved spatial resolution arc able to separate deep activity from the propagated activity in superficial cortical areas.

Additional problems are caused by the deviation of dipole orientation. MEG is more sensitive to tangential sources than to radial sources. In patients undergoing presurgical evaluation Cohen et al. (1990) produced well defined dipoles with a predominant radial component by passing a weak current pulse through the depth electrodes. The localizations error ranged from 24 to $40 \mathrm{~mm}$ for deep sources in the amygdala or the mid temporal gyrus (Cohen et al., 1990). Simulation studies showed that the localization error increases with increasing deviation of dipole orientation from a tangential towards a radial orientation (Menninghaus et al., 1994; Menninghaus and Lütkenhöner. 1995). Recause little is known about the dipole orientation of electromagnetic fields generated in the temporal lobe, especially in the temporomesial structures, there is no possibility to estimate the influence of dipole orientation under the registration conditions of this study.

\subsection{The head model}

The assumption of a spherical head model will also cause an error in localizing the dipole source, although this kind of error is expected to be a certain aberration from the true solution rather than the lack of, plausible, solutions as we found in the majority of our patients. Even if the effects of 
local variations in skull and scalp thickness more affects the localization accuracy of EEG signals rather than of MEG signals (Cuffin, 1993), the error in source localization for MEG signals increases with increasing depth of the source using a spherical head model (Menninghaus et al., 1994; Menninghaus and Lütkenhöner, 1995). Up to now, the advantage of realistic head models has been demonstrated for a maximal depth of $3 \mathrm{~cm}$ (Menninghaus et al., 1994).

\section{Conclusions}

This study shows the advantages of the application of methohexital for MEG recordings, since the simple and safe short narcosis considerably increases the spike density. Thus, registration periods are shortened and movement artifacts are reduced. With respect to the identification of the primary epileptogenic area, MEG recorded spikes after methohexital application allow a correct lateralization of the epileptogenic area in about 2 thirds of the patients with TLE, whereas the localizing value is still unsatisfactory. The results of this study indicate that. under these measurement conditions, the MEG recorded spikes predominantly reflect the neuronal activity generated in temporolateral structures, whereas the activity of deeper sources, especially of temporomesial structures, is obviously difficult to register using the MEG. In order to improve measurement conditions. whole-head systems are a possible solution. Further progress in localization algorithms and the use of realistic head models are strongly requested. In order to explain the differences in peak latencies observed in our investigation. simultancous recordings of ECoG and MEG are essential. An improved knowledge about the source configuration corresponding to epileptiform activity and about propagation mechanisms is required prior to a revised assessment of the clinical relevance of $\mathrm{MEG}$ recordings for the presurgical evaluation of epilepsy patients.

\section{Acknowledgements}

This research was supported by grants from the Deutsche Forschungsgemeinschaft (Klinische Forschergruppe Biomagnetismus and Biosignalanalyse, Ho 847/6). We thank our neurosurgical colleagues J. Schramm, M.D., J. Zentner. M.D.. D. Van Roost, M.D., and E. Behrens, M.D., (Bonn) who implanted the subdural and intrahippocampal depth electrodes, and who performed the temporal lobe resections. Furthermore, we want to thank the anaesthesiological colleagues M. Möllmann, M.D. N. Mertes, M.D., L. Spinne, M.D., G. Westermeier, M.D., C. Goeters, M.D., M. Reuss. M.D., W. Frebel..M.D. and L. Ruta. M.D. (Münster), who performed the methohexital short narcosis, and our epileptological colleagues A. Barbori, M.D., and S. Gass, M.D (Bonn), who look care for the patients during the presurgical evaluation and the $\mathrm{MEG}$ recordings.

\section{References}

Aasley. J., Silfvenius, H. and Zetterlund, B. Barbiturate elfects on EEG abnormality in complex partial epilepsy. J. Neurol., 1989. 236: 15-20. Ahonen, A.I.. Hämäläinen. M.S.. Ilmoniemi, R.J., Kajola. M.J.. Knuutili, J.E.T., Simola, J.T. and Vilkman, V.A. Sampling theory for neuromag netic delector arrays. IEEE Trans. Biomed. Eng.. 1993, 40: 859-869.

Alarcon, G., Guy. C.N., Binnie, C.D., Wilker, S.R., Elwes, R.D.C. and Polkey. C.E. Intracerebral propagation of interictal activity in partial epilepsy: implications for source localization. J. Neurol. Neurosurg. Psychialry. 1994. 57: 435-449.

American Electroencephalographic Society guidelines for stindard elestrode pusition nomenclature. J. Clin. Neurophysiol., 1991, 8: 200223 .

Bickford. R., Falconer. J., Sem-Jacobien, C., Dodge, H. and Schnugg. F. Some eftects of barbiturate andesthesia on the depth electrogram. Mavo Clin. Proc.. 1953. 28: 162-165.

Brazier. M. Prenarcotic doses of harbiturates ats an aid in tocaljoing diseased brain tissue. Anaesthesiology. 1969. 31:78-8.3.

Brockhaus, A., Hufnagel. A.. Nadstawek, J., Eheling, B.J.. Van Roost, D. and Elger. C.E. Activation of epileptogenic foci by thiopental in electrocorticographic recordings with subdural strip electrodes and intrahippocanpal depth electrodes. J. Epilepsy. 1995, 8: 153163.

Celesia. G.G. and Paulsen. R.E. Electroencephalographic actuvation with sleep and methohexital. Arch. Neurol., 1972. 27: 361-36.3.

Cohen, D., Cuffin. B.N., Yunokuchi, K., Maniewski. R., Purcell. C.. Cosgrove. G.R., Ives. J.. Kennedy. J.G. and Schomer. D.L. MEG versus EEG localization test using implinted sourcen in the buntan brain. Ann. Neurol. 1990. 28: 811-817.

Cuffin. N. Effects of local variations in skull and sealp thickness on EEGs and MFGs. IEEE Trans. Biomed. Eng. 1993, 40: 42-48.

Dasheiff. R. and Kofke. W. Evaluation of the thiopental test in epilepsy surgery patients. Epilepsy Res.. 1993. 15: 253-258

Ehersole, J.S.. Squires. K.C., Eliashiv, S.D. and Smith, J.R. Application of magnetic source imaging in evaluation of candidates for apilepsy surgery. Funct. Neuroimaging, 1995, 5: 267-288.

Elger. C.E. and Speckman. E.J. Focal interictal epileptiform discharges (FIED) in the epicortical EEG and their relations to spinal potentials in the rat. Electroenceph. clin. Neurophysiol. 1980), 48; 447-460)

Elger. C.E. Facts in epileptology which are of porsitele relevance for biomagnetism. In: M. Hoke. S.N. Erné, Y.C. Okada and G.L. Romani, (Eds). Biomagnetism: Clinical Aspects. Excerplat Medical. Amsterdam. 1992. pp. 53-60).

Engel. J., Jr. (Ed.). Surgical Treatment of the Fpilepsies, Raven Press. New York, 1993.

Ford. E.W.. Morrell. F. and Whisler. W.W. Methohexilal anaesthesia in the surgical treatment of uncontrollable cpilepsy. Anesthes. Analg. 1982, 1: 997-1001.

Fuster. B. Gibbs, E. and Gibbs, F. Pentothal sleep as an aid to the diagnowis and localization of seizure discharges of the psychomotor type. Dis. Nerv. Syct.. 1948, 7: 199-202

Gharih. S. Sulherling. W.W. Nakasiato. N. Barth. D.S.. Baumgartner. C. Alexopoulos, N.. Taylor, S. and Rogers. R.L. MEG and ECOG focalization accuricy test. Electroenceph. clin. Neurophysiol. 1995. 94: 109114.

Gumpert, J. and Paul, R. Activation of the electroencephalogram with intravenous brevitol (methohexitonel: the lindings in 100 cakes. J. Neurol. Neurosurg. Psychiatry. 1971. 34: 646-648

Harris, R. and Paul. R. The use of methohexitone in clectrocorticography. Electroenceph. clin. Neurophysiol. 1969. 27: 3.33-334

Hazeaux, C. Tisserant. D.. Vespignani. H.. Hummer-Sigiel, M., KwanNing. V. and Laxenaire, M.C. Retentissement électroenéphalegraphique de l'anesthésic au propofol. Ann. Fr. Aneshl. Reanim.. 1987. 6: 261266.

Hutnagel. A.. Elger. C.. Boeker. D.. Linke. D.. Kurthen, M. and Solymosi, L. Activation of the epileptogenic focus during intracirotid amobarbital 
test. Electrocorticographic registration via subdural electrodes. Electroenceph. clin. Neurophysiol., 1990a, 75: 453-463.

Hufnagel, A., Elger, C., Nadstawek, J., Stoeckel, H., Boeker, D. Specific response of the epileptogenic focus to anaesthesia with propofol. J. Epilepsy, 1990b, 3: 37-45.

Hufnagel, A., Burr, W., Elger, C.E., Nadstawek, J. and Hefner, G. Localization of the epiletogenic focus during methohexital-induced anesthesia. Epilepsia, 1992, 33: 271-284

Kiersey. D., Bickford. R. and Faulconer. A. Flectrnencephalographic patterns produced by thiopental sodium during surgical operations: Description and classification. Br. J. Anaesthesiol,, 1951, 23: 141-152.

Kowalik, A.. Hufnagel, A. and Elger, C.E. Methohexital for the localization of the epileptogenic focus. Retest reliability in a study with simultaneous depth and subdural electrodes. J. Epilepsy, 1997, in press.

Lieb. J., Sclabassi, R., Crandal, P. and Buchness, R. Comparison of the action of diazepam and phenobarbital using EEG-derived power spectra obtained from temporal lobe epileptics. Neuropharmacology, 1974. 13: 769-783.

Lieb, J., Babb, T. and Engel, J. Quantitative comparison of cell loss and thiopental-induced EEG changes in human epileptic hippocampus. Epilepsia 1989, 30: 147-156.

Lüders, H. and Awad. I. Conceptual considerations. In: H. Luiders (Ed.), Epilepsy Surgery, Raven Press, New York, 1991, pp. 51-62

Luiders, H.O., Engel, J. and Munari. C. General principles. In: J. Engel, Jr. (Ed.), Surgical Treatment of the Epilepsies, 2nd edn., Raven Press, New York, 1993, pp 137-153.

Lütkenhöner, B. A simulation study of the resolving power of the biomagnetic inverse procedure. Clin. Phys. Physiol. Meas., 1991, 12 (Suppl A): 73-78

Lütkenhöner, B., Lehnertz, K., Hoke, M. and Pantev, C. On the biomagnetic inverse problem in the case of multiple dipoles. Acta Otholaryngol. Suppl. Stockh., 1991, 491: 94-104.

Lütkenhöner, B., Elbert, E.. Menninghaus, E., Steinsträter, O., Wienbruch, C. Electro- and magnetoencephalographic source analysis: current status and future requirements. In: H.J. Herrmann, D.E. Wolf and E. Pöppel (Eds), Workshop on Supercomputing in Brain Research: From Topography to Neural Networks. World Scientific Publishing, 1995, pp. 175192.

Menninghaus, E. and Lütkenhöner, B. How silent are deep and radial sources in neuromagnetic measurements? In: C. Baumgartner, L. Deecke. G. Stroink and S. Williamson (Eds.), Biomagnetism: Fundamental Research and Clinical Applications, Studies in Applied Electromagnetics and Mechanics. Vol. 7. Elsevier. Amsterdam. 1995, pp. 352356.

Menninghaus, E., Lütkenhöner, B. and Gonzales. S.L. Localization of a dipolar source in a skull phantom: realistic versus spherical model. IEEE Trans. Biomed. Eng., 1994, 41: 986-989.

de Munck, J.C., Vijn, P.C. and Lopes da Silva, F.H. A random dipole model for spontaneous brain activity. IEEE Trans. Biomed. Eng.. 1992, 39: 791-804.

Musella. L., Wilder, B.J. and Schmidt, R.P. EEG activation with intravenous brevital in psychomotor epilepsy. Neurology, 1969. 19: 278.

Nakasato. N., Levesque, M.F., Barth. D.S., Baumgartner, C., Rogers, R.L. and Sutherling, W.W. Comparison of MEG, EEG, and ECoG source localization in neocortical partial epilepsy in humans. Electroenceph. clin. Neurophysiol., 1994, 71: 171-178.

Nunez, P.L. The brain's magnetic field: some effects of multiple sources on localization methods. Electroenceph. clin. Neurophysiol.. 1986, 63: $75-82$.

Ogura, Y. and Sekihara, K. Relationship between dipole parameter estimation errors and measurement conditions in magnetoencephalography. IEEE Trans. Biomed. Eng., 1993, 40: 919-924.

Pampiglione, G. Induced fast activity in the EEG as an aid in the localization of cerebral lesions. Electroenceph. clin. Ncurophysiol., 1952. 1: $79-82$.

Pantev, C., Gallen. C., Hampson, S., Buchanan, S. and Sobel. D. Reproducability and validity of neuromagnetic source localization using a large array biomagnetometer. Am. J. EEG Tcchnol., 1991, 31: 83101.

Pateau, R., Hämäläinen. M.. Hari, R., Kajola, M., Karhu, J, Larsen, T.A., Lindahl, E. and Salonen, O. Magnetoencephalographic evaluation of children and adults with intractable epilepsy. Epilepsia, 1994, 35: $275-284$.

Petsche, H.. Rappelsberger, P.. Lapins. R. and Vollmer, R. Rhythmicity in seizure pattern: topographical aspects. In: E.J. Speckman and H. Caspers (Eds.), Origin of Cerebral Field Potentials. Thieme, Stuttgart, 1969. pp. $60-79$.

Scherg. M. Fundamentals of dipole source potential analysis. In: F. Grandori, M. Hoke and G.L. Romani (Eds.), Auditory Evoked Magnetic Fields and Potentials. Advances in Audiology. Vol 6., Karger, Basel. 1994. pp. 40-69.

Scherg, M. and Ebersole, J.S. Brain source imaging of focal and multifocal epileptiform EEG activity. Neurophysiol. Clin., 1994. 24: 51-60.

Smith. R.J., Schwartz., B.J., Gallen, C., Orrison, W., Lewine, J., Murro, A.J., King, D.W and Park, Y.D. Utilization of multichannel magnetoencephalography in the guidance of ablative seizure surgery. J. Epilepsy 1995, 8: 119-130.

Sperling, M.R. Brown, W.J. and Crandall, P. Focal burst-suppression induced by thiopental. Electroenceph. clin. Neurophysiol., 1986, 63: 203-208.

Stefan, H., Schneider, S., Abraham-Fuchs, K., Bauer, J., Feistel, H., Pawlik, G., Neugebauer, U., Röhrlein, G. and Huk, W.J. Magnetic source localization in focal epilepsy. Brain 1990, 113: 1347-1359.

Stefan, H., Schüler, P., Abraham-Fuchs, K.. Schneider, S., Gebhardt, M. Neubauer. U., Hummel, C., Huk, W.J. and Thierauf, P. Magnetic source localization and morphological changes in temporal lobe epilepsy: comparison of MEG/EEG, ECoG and volumetric MRI in presurgical evaluation of operated patients. Acta Neurol. Scand. Suppl., 1994. 152: $83-$ 88.

Supek. S. and Aine. C.J. Simulation studies of multiple dipole neuromagnetic source localization: model order and limits of source resolutions. IEEE Trans. Biomed. Eng., 1993, 40: 529-540.

Tan, S., Roth, B.J. and Wiskow, J.P. The magnetic field of cortical current sources: the application of a spatial filtering model to the forward and inverse problem. Electroenceph. clin. Neurophysiol,, 1990, 65: 344 360 .

Tiihonen, J., Hari, R., Kajola, M., Nousiainen, U. and Vapalahti, M. Localization of epileptic foci using a large-area magnetometer and functional brain anatomy. Ann. Neurol., 1990, 27: 283-290.

Wilder, B. Electroencephalogram activation in medically intractable epileptic patients. Arch. Neurol., 1971. 25: 415-426.

Wyler, A.R.. Richey, E.T., Atkinson, R.A. and Hermann, B.P. Methohexital activation of epileptogenic foci during acute electroencephalography. Epilepsia, 1987, 28: 490-494. 